\title{
MRI PRESENTATION OF FEMALE GENITAL TUBERCULOSIS: A CASE REPORT
}

\author{
Ana GAZIKALOVIĆ ${ }^{1 *}$, Oto AĐIĆ \\ ${ }^{1}$ Mother and Child Health Care \\ Institute of Serbia "Dr Vukan Čupić" \\ Belgrade, Serbia, ${ }^{2}$ Centre for Imaging \\ Diagnostics Oncology Institute of \\ Vojvodina, Sremska Kamenica, Serbia
}

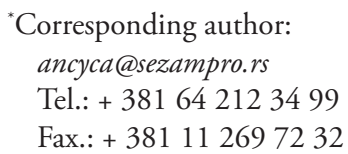

Received: November 11, 2015

Accepted: January 12, 2016

Key words: Tuberculosis - Female genital tract - Magnetic resonance imaging.

\begin{abstract}
Objective - To present a rare case of female genital tuberculosis and its appearance on magnetic resonance imaging. Case report - A sixteen year old girl, presenting with appetite and weight loss, pelvic pain, headache, shortness of breath and secondary amenorrhea, was admitted to hospital with a suspected ovarian tumor. Clinical examination, laboratory analysis and radiological procedures were performed. Chest X-ray showed minimal pleural effusion on the left hand side. Voiding cystourethrography and intravenous urography were without abnormalities. Abdominal and pelvic ultrasound examination revealed the presence of an ovarian mass and ascites. Abdominal MRI showed mesenteric lymphadenomegaly, ascites with peritoneal nodular implants that showed diffusion restriction and post-contrast enhancement. Pelvic MRI showed heterogeneous masses involving both ovaries, free fluid in the cul-de-sac, nodular peritoneal implants with diffusion restriction and pathological post-contrast enhancement. Cytological analysis of the peritoneal fluid was undertaken. Laparoscopy and pathohistological analysis of the peritoneal specimen obtained were performed. Genital tuberculosis was confirmed. Conclusion - In this case it is stressed that even though MRI is the most sensitive diagnostic procedure, it is not sufficiently specific. The correct diagnosis was established by pathohistological examination. Adequate diagnosis and treatment are essential because the untreated disease causes severe morbidity, including sterility.
\end{abstract}

\section{Introduction}

Tuberculosis (TB) represents a major health problem in the world and is a major cause of morbidity and mortality in both immuno-competent and immuno-compromised individuals $(1,2)$. The genitourinary tract is the most common site of extrapulmonary involvement of TB (3). Female genital TB is a chronic disease, with non-specific clinical, laboratory and radiological findings, which makes it difficult to diagnose. It mostly affects the Fallopian tubes, and spreads to the ovaries forming a tubo-ovarian mass (4).
The clinical symptoms and signs of genital TB may mimic peritoneal carcinomatosis or ovarian malignancies, which makes them difficult to differentiate (1). Radiological presentation of ovarian TB is not specific and cannot be distinguished from peritoneal dissemination of malignant tumors or ovarian carcinomas $(3,4,5)$.

This case report shows that TB has unspecific presentation on MRI, and that it should be included in the differential diagnosis of tumor-like pelvic masses. 


\section{Case report}

A sixteen year old girl, with poor appetite and $16 \mathrm{~kg}$ weight loss, headache, shortness of breath, pelvic pain and secondary amenorrhea, was admitted to our Institute with a suspected ovarian tumor. The girl was referred to our Institute from the primary health care service, where the clinical symptoms and signs indicated a pelvic - ovarian tumor. Her clinical history was unremarkable, her red blood and white blood cell counts were within the normal ranges, and she had not been significantly ill before. In the laboratory analysis, high values of CRP were found (CRP; $40.7 \mathrm{mg} / \mathrm{l})$; hemo-culture and urinoculture were sterile; ELISA and HIV tests were negative; HBs and anti HCV antigens were negative; ANA was negative, and Ca 125 tumour markers were mildly elevated, at $57 \mathrm{U} / \mathrm{ml}$. Heart ultrasound (US) and eye examinations were without abnormalities. Chest ra-

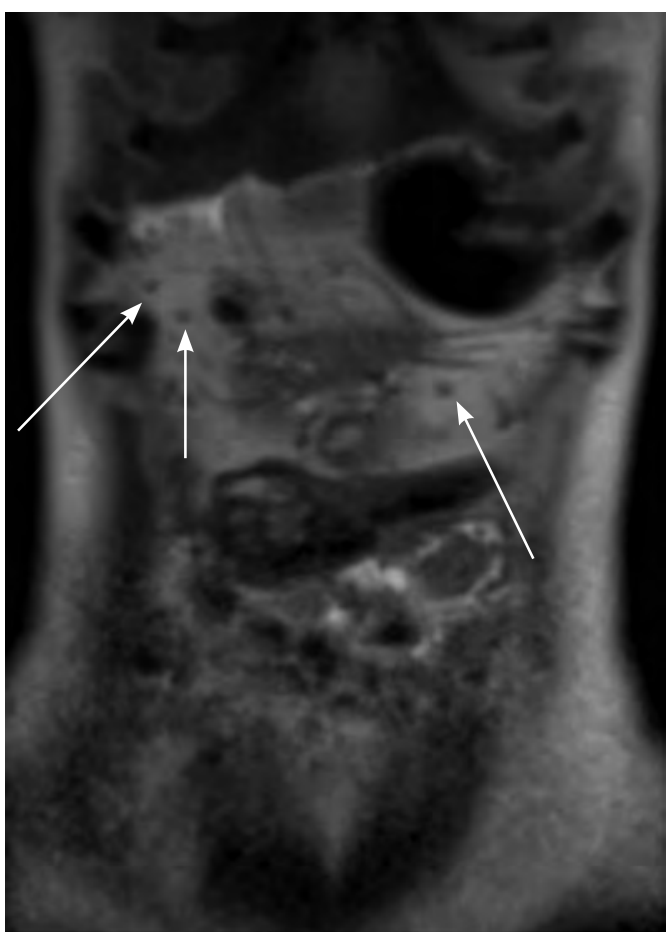

Fig. 1 Magnetic resonance (T2W coronal) showing hypointense peritoneal nodules (arrows) and a small amount of free peritoneal fluid. diography showed minimal pleural effusion on the left-hand side. Voiding cystourethrogram and intravenous urography were without abnormalities. Abdominal US showed a large amount of free peritoneal fluid. Pelvic US showed the heterogeneous appearance of both ovaries and a large amount of free inhomogeneous peritoneal fluid. Abdominal and pelvic MRI showed free peritoneal fluid, with peritoneal nodular implants (Fig. 1) diffusion restriction and pathological post-contrast enhancement (Fig. 2).

The mesenteric lymph nodes (LN) were enlarged. Both ovaries were enlarged, with heterogeneously low signal intensity (SI) on T2 weighted sequences (W), without normal follicular structure and morphology (Fig. 3). The ovarian masses and free peritoneal fluid, low in SI on T2W (Fig. 3), showed diffusion restriction, and there was intense heterogeneous post-contrast enhancement of the ovarian masses and peritoneal wall (Fig. 4). Enlarged para iliac, presacral, and ischiorectal LN were found, as well as multiple small perirectal LN.

The findings were described as bilateral ovarian neoplasms, with peritoneal and lymphogenic dissemination. Explorative laparoscopy and peritoneal biopsy showed no tumor cells in the peritoneal fluid. The histopathological report mentioned "multiple chronic inflammatory granulomas with central caseous necrosis surrounded by epithelioid histiocytes and Langerhans cells. No acid-resist-

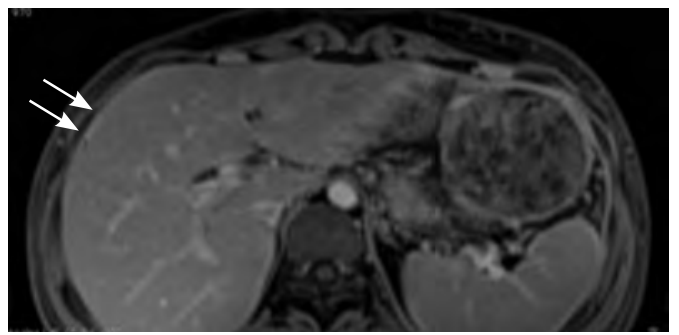

Fig. 2 Magnetic resonance (T1W FS post contrast axial) showing hepatic capsule and peritoneal enhancement 120 seconds after intravenous administration of paramagnetic contrast medium. 
ing bacilli were found in the granulomas". The method used to detect TB bacilli was the Ziehl-Neelsen stain method. Although Mycobacterium tuberculosis was not found in the specimen, anti-TB treatment was indicated. After starting the anti-tuberculosis treatment, the girl recovered and was released home with a regimen of anti-TB combination therapy.

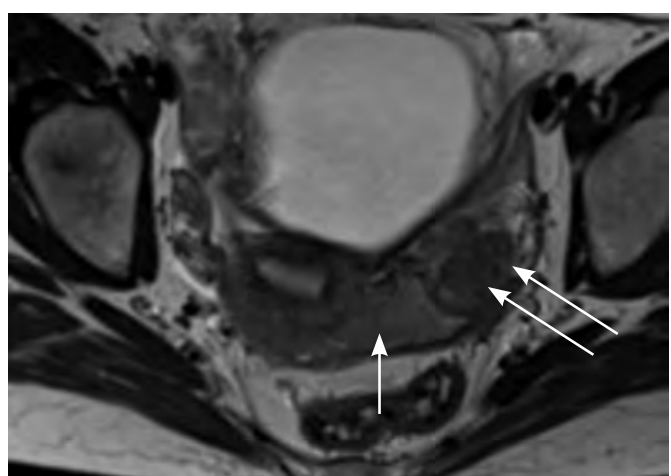

Fig. 3 Pelvic magnetic resonance imaging (T2W axial sequence) showing a hypointense $T 2 W$ lesion on the left ovary (arrows) and hypointense T2W peritoneal fluid (single arrow).

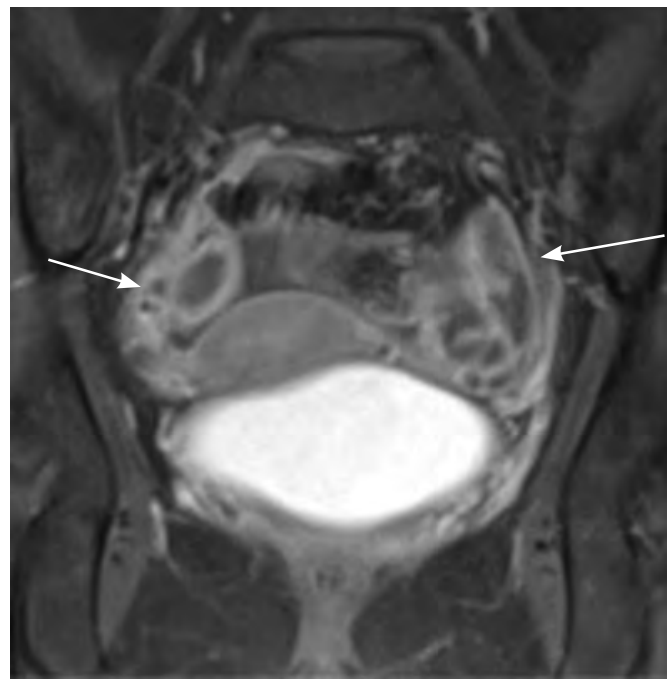

Fig. 4 Pelvic magnetic resonance imaging (T1W FS coronal sequence) showing heterogeneous enhancement of the ovarian lesions (arrows).

\section{Discussion}

Female genital TB is a chronic disease with nonspecific symptoms. It develops as the result of pulmonary, urinary, intestinal or bone hematogenous or lymphatic dissemination of TB (6). The Fallopian tubes are mostly affected, followed by the endometrium, ovaries, vagina, vulva and cervix (2). Infection of the Fallopian tubes and endometrium can result in sterility (7). The most common clinical symptoms are abdominal distension, pelvic tumors and ascites, which are often interpreted as peritoneal carcinomatosis or ovarian malignancy (7).

Typical histological findings include epitheloid cellular granulomas with or without giant Langerhans cells. Caseous necrosis is rare in samples from the female genital tract (6). Also, the triplex- polymerase chain reaction (PCR) technique can be used to differentiate between mycobacterium tuberculosis and non-tuberculous mycobacteria from formalin fixed, paraffin embedded tissue (6).

Ovarian TB is mostly a sequel of the tuber disease and presents as a tubo-ovarian abscess (TOA). In the TOA complex, adnexal masses are present as well as a large amount of peritoneal fluid. Ovarian masses may have solid and cystic components, sometimes with calcifications, and are often associated with ascites, omental and mesenteric infiltrates and peritoneal thickening (4). There is often multiple LN enlargement and necrosis, presented as peripheral enhancement and central non-enhanced areas. Loculated peritoneal fluid collections may also be found (4). Tuberculous peritonitis is seen in association with other forms of gastrointestinal TB. Three types of TB peritonitis have been described. The wet type, seen in most patients, is characterized by a large amount of viscous fluid. The fibrotic fixed type consists of large omental masses and sometimes, loculated ascites. The dry or plastic type is characterized 
by caseous nodules, fibrous peritoneal reaction and dense adhesions $(2,5,8,9)$.

For diagnosis of genital TB, many radiological diagnostic procedures are used. However, ionizing radiation in children should be avoided, therefore US and MRI are predominantly used. On US, ovarian masses have both solid and cystic components and TOA may calcify (which can suggest TB). In addition, a thickened omentum and a large amount of ascites may be seen $(2,4)$. MRI may demonstrate thickened salpinges or nodularities along tubo-ovarian surfaces. The walls of tuberculous TOAs are irregular, with a serrated or nodular inner wall that has low SI on T2W images, and is often accompanied by nodular thickening of the peritoneum as a sign of peritonitis. The SI of TOA varies depending on the viscosity and protein content. In the late phase, commonly there may be dense adhesions with the uterus and other adjacent organs (4). However, all the diagnostic procedures previously mentioned are not specific enough to diagnose TB. Extensive abdomino-pelvic TB with peritoneal spread may simulate disseminated ovarian carcinoma, both clinically and on imaging methods (4).

In our case, the clinical symptoms and signs (pelvic pain, weight loss) and laboratory analysis (mildly elevated Ca 125) indicated ovarian carcinoma. The patient's MRI examination presented bilateral tubo-ovarian masses which is more a characteristic of $\mathrm{TB}$ (TOA) and less probably ovarian carcinoma. All these findings correlated with the literature data $(2,3,4,5,8,9)$. Definitive diagnosis is based on finding the TB bacilli in a biological sample, or cell cultures grown on Lowenstein medium. In addition, molecular diagnosis with PCR is very useful in culture negative cases. It is necessary to make a correct diagnosis, since treatment of TB and carcinoma differs significantly (6).
Treatment includes a combination of anti-tuberculous drugs: pyrazinamid, streptomycin, para-aminosalicylic acid, isoniazid, rifampicin and ethambutol. A four-drug regimen consisting of pyrazinamid, isoniazid, rifampicin and ethambutol is used for the first two months, followed by triple or dual therapy. The total duration of treatment should be six months to a year (7). Surgical procedures are undertaken, if there is a persistent pelvic mass and pain, or persistent bleeding after nine months of treatment (10).

\section{Conclusion}

Female genital TB should be included in the differential diagnosis of solid and cystic tuboovarian lesions, since its incidence is rising in developed and developing countries. The fallopian tubes are affected most often, and in many patients the symptoms point to ovarian cancer. Many radiological procedures are used to diagnose genital TB, of which MRI is highly sensitive, but not specific. Definitive diagnosis is only established by the detection of Koch bacilli or histological analysis of the biopsy material. Establishing the diagnosis of the disease on time is important, since delayed treatment is associated with severe morbidity.

Authors' contributions: Conception and design: AG, $\mathrm{OA}$; Acquisition, analysis and interpretation of data: AG, OA; Drafting the article: AG, OA; Revising it critically for important intellectual content: AG, OA.

Conflict of interest: The authors declare that they have no conflict of interest.

\section{References}

1. Yassaee F, Farzaneh F. Familial tuberculosis mimicking advanced ovarian cancer. Infect Dis Obstet Gynecol. 2009;2009:736018.

2. Vanhoenacker FM, De Backer AI, Op de Beeck B, Maes M, Van Altena R, Van Beckevoort D, et al. Imaging of Gastrointestinal and Abdominal Tuberculosis. Eur Radiol. 2004;14 (Suppl 3):E103-15. 
3. Jung YY, Kim JK, Cho KS. Genitourinary Tuberculosis: Comprehensive Cross-Sectional Imaging. AJR 2005;184:143-50.

4. Kim SH, Kim SH, Yang DM, Kimm KA. Unusual Causes of Tubo-ovarian Abscess: CT and MR Imaging Findings. RadioGraphics 2004;24:1575-89.

5. Harisinghani MG, McCloud TC, Shepard JO, Ko JP, Shroff MM, Mueller PR. Tuberculosis from Head to Toe. RadioGraphics 2000;20:449-70.

6. Mondal SK, Dutta TK. A Ten year Clinicopathological Study of Female Genital Tuberculosis and Impact on Fertility. J Nepal Med Assoc. 2009;48(173):52-7.
7. Botha MH, Van der Merwe FH. Female Genital Tuberculosis. SA Fam Pract 2008;50(5):12-6.

8. Engin G, Acunas B, Acunas G, Tunaci M. Imaging of Extrapulmonary Tuberculosis. RadioGraphics 2000;20:471-88.

9. Burrill J, Williams CJ, Bain G, Conder G, Hine AL, Misra RR. Tuberculosis: A Radiologic Review. RadioGraphics 2007;27:1255-273.

10. Gupta S, Tomer G. Pelvic-Peritoneal Tuberculosis Mimicking Ovarian Malignancy: A Case Report. WebmedCentral OBSTETRICS AND GYNAECOLOGY2011;2(1):WMC001520. [cited 2015 Nov 25] Available from: http://www. webmedcentral.com/article_view/1520. 